\title{
Feux croisés sur les arts des îles Salomon
}

À propos de deux expositions récentes et de leur catalogue

\section{Gilles Bounoure}

\section{(2) OpenEdition Journals}

Édition électronique

URL : http://journals.openedition.org/jso/7383

DOI : $10.4000 /$ jso. 7383

ISSN : $1760-7256$

Éditeur

Société des océanistes

Édition imprimée

Date de publication : 15 décembre 2015

Pagination : 337-348

ISBN : 978-2-85430-126-7

ISSN : 0300-953x

Référence électronique

Gilles Bounoure, «Feux croisés sur les arts des îles Salomon », Journal de la Société des Océanistes [En ligne], 141 | juillet-décembre 2015, mis en ligne le 15 décembre 2018, consulté le 02 mai 2019. URL: http://journals.openedition.org/jso/7383 ; DOI : 10.4000/jso.7383 


\section{MISCELLANÉES}

\section{Feux croisés sur les arts des îles Salomon. À propos de deux expositions récentes et de leur catalogue}

par

\section{Gilles BOUNOURE*}
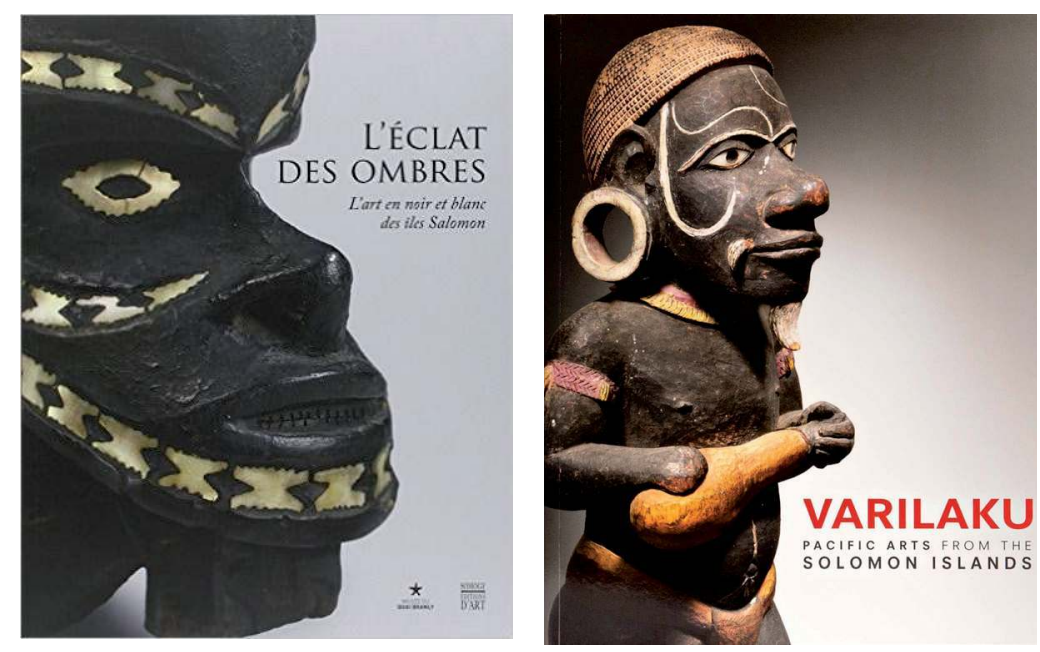

ces zones pourraient aider à concevoir un panorama plus complet de ces expressions artistiques, demeurées méconnues dans leur détail et énigmatiques pour beaucoup d'entre elles. À côté de sources anciennes à mieux exploiter, ce panorama aurait à prendre en compte les préoccupations des Salomonais d'aujourd'hui dont aucune de ces manifestations n'a tenté de se faire l'écho, pour de probables raisons de prudence à mesurer elles aussi.

À Canberra et à Paris, le public aura pu voir à peu d'années d'intervalle deux expositions importantes sur les arts anciens des îles Salomon, entreprises notables en raison du peu de manifestations consacrées jusqu'à présent à cet ensemble de cultures, mais aussi du fait de leurs mérites respectifs qu'il importait de relever. Croisant leurs faisceaux à la façon des phares côtiers et se différenciant comme eux par leur secteur angulaire et leur portée, l'une et l'autre auront marqué leurs zones propres d'ombres et de lumières. Une fois délimitées et mises bout à bout,

\section{Art ou arts?}

De ces deux expositions, "Varilaku : Pacific arts from the Solomon Islands", présentée à la National Gallery of Australia du 24 février au 29 mai 2011, et "L'éclat des ombres. L'art en noir et blanc des îles Salomon ", au musée du quai Branly du 18 novembre 2014 au $1^{\text {er }}$ février 2015 , le rédacteur de ces lignes n'a pu voir que la seconde, très ingénieusement disposée dans l'espace contraignant de la "Mezzanine Est » de ce bâtiment ${ }^{1}$. Pour celle de Canberra dont ne sera

1. Le plan de l'exposition est détaillé, vitrine par vitrine, dans la « liste des œuvres exposées » (pp. 211-216) du catalogue parisien. * gillesbounoure@gmail.com 
décrit ici que le catalogue, la presse australienne a livré d'assez longs comptes rendus (par exemple sous les plumes de Siobhan Heanue et d'Helen Musa, 2011), soulignant que c'était la première manifestation jamais consacrée à ces arts dans un musée de ce pays, commentaire valant aussi pour l'exposition parisienne. Si l'on excepte la présentation permanente du British Museum "The Solomon Islanders" conçue par Bryan A.L. Cranstone et Dorota Starzecka, visible du 31 mai 1974 au 23 juin 1985, avec une publication qui fut longtemps la seule en ce domaine (Starzecka 1974), ces arts n'ont donné lieu qu’à un très petit nombre d'initiatives d'institutions publiques.

Parmi elles on citera le Burke Museum de Seattle présentant entre août 2008 et juillet 2009 sous le titre "From the Solomon Islands 1928-30» la petite collection récoltée sur place par l'ornithologue Walter J. Eyerdam, membre de la Whitney South Seas Expedition lancée par l'American Museum of Natural History, léguée au musée par ses descendants, le musée national d'Honiara renouvelant ses vitrines et son parcours à l'occasion du $11^{\mathrm{e}}$ Festival of Pacific Arts de 2012 et y ajoutant en 2014 une exposition intitulée "Black Birding. The Queensland-Pacific Indentured Labour Trade and Australian South Sea Islanders", l'Ayers House Museum d'Adelaide organisant au printemps 2013, sous le titre "Art Treasures from Solomon Islands" une exposition réunissant les membres de l'Artists'Association of Solomon Islands constituée à la faveur du festival précité, initiatives récentes que complètent les expositions de Canberra et de Paris évoquées ici.

Elles se distinguent d'abord par leur titre, le premier paraissant annoncer des arts divers dont le second semble au contraire affirmer l'unité, même si l'un et l'autre mêlent habilement le pluriel et le singulier. Indépendamment des soucis de communication ayant pu présider à leur choix, cette divergence traduit une difficulté presque constante dans l'appréhension, l'analyse et la présentation des arts océaniens, mélanésiens surtout. D'une proximité ou d'une contiguïté géographique et d'une histoire coloniale largement partagée, n'est-ce pas une pétition de principe que de déduire une homogénéité d'expression artistique entre des sociétés souvent en conflit les unes avec les autres et dont beaucoup d'objets marquaient la singularité ? Dans le cas des îles Salomon, où les premiers découvreurs espagnols étaient venus chercher les bords d'un continent inconnu avant d'en explorer les détroits et d'y reconnaître un archipel, ne seraitce pas revenir à la même illusion que de vouloir y trouver une continuité artistique dont font douter aussi bien les distances que des disparités de langue, de mœurs et de style observables parfois à l'intérieur d'une même île ?
Il n'est pas inutile d'examiner comment les meilleurs spécialistes des décennies passées ont abordé cette difficulté, se référant souvent à une partition entre trois aires stylistiques déjà classique en leur temps, et sur laquelle on reviendra. Selon Tibor Bodrogi (1959 : 22-23) :

"the Solomon Islands [...] form a separate style province [with] three cultural districts. Well-developed initiation rites are the salient feature of the western group, head hunting is characteristic of the central islands, while totemism and the belief in powerful marine spirits is typical of the eastern islands. Each of the different cultural traits has given rise to corresponding works of art and caused the development of different forms and functions; nevertheless, the use of identical media and the ressemblance between the decorative elements lends a uniform character to the art of the Solomon Islands. "

Alfred Bühler (1962: 142 et 147) écrivait d'abord :

«Malgré les différences culturelles profondes, les formes d'art de ce groupe d'îles peuvent être comprises en une seule province à style caractéristique. "

Mais il concluait à quelques pages de distance :

«Les îles Salomon constituent une région sans unité stylistique. »

En quelques mots, Gerd Koch (1969 : 87) formulait l'hypothèse d'une diversification des arts de l'archipel au cours des âges :

"eine Vielfalt unterschiedlicher Lokalkulturen existierte, die jedoch offensichtlich von einer gemeinsamen Basis entwickelt waren. "

Carl A. Schmitz (1971 : 249 et 251) estimait plus simplement : "apart from [some] variations, the entire area exhibits the same basic stylistic features", autorisant à parler de "basic Solomon Islands style ». Pour D. Starckeza (1974 : 40-42),

" the great cultural diversity of the Solomons is reflected in the art of the islands but there are certain underlying similarities of style and decorative motifs. Certain characteristics are common to the whole region: both two-dimensional and three-dimensional représentations occur; large figure are relatively inusual; the importance of the head in human figure representations is emphasised by its size or its style [...]. Solomon Islands art has an inusually highly developed sense of form and combines vigour and intensity with elegance..."

\section{Aux yeux de Douglas Newton (1979 : 50) :}

" the first impression given by Solomon Islands art is that it is dramatic, elegant and curiously luxurious. This is due mainly to the predominance of black as an overall color, with very minor highlights in red and small linear patterns in white. " 
À ceux de Waldemar Stöhr (1987 : 205) :

"Die Kunst der Solomonen hat [...] ein ziemlich einheitliches Gepräge",

c'est-à-dire un caractère relativement homogène. Plus récemment, Christian Kaufmann (1993: 559) en traitait lui aussi comme d'une unité :

"L'art des îles Salomon (y compris celui des îles Santa Cruz) est dominé par le contraste entre une forme proche de la vie, très élaborée, et une "dénaturation" des surfaces par un fin revêtement d'une couleur noire à base de résine, souvent incrusté de fragments de nacre. »

Enfin, pour Deborah Waite (2011:34), l'unité de ces arts tiendrait à l'omniprésence de la figuration humaine et de son « langage corporel " :

" Here, all the images - or figures - can be described as anthropomorphic in that they have human-like heads, limbs and torsos, but exactly what they represent varies. Some may connote the presence of ancestral or non-human spirits. Other are intended to capture the essence of living people. And one should be able to tell the difference. A process of visual coding has long been at work among artists throughout the Solomon Islands. "

Ni la similarité de matériaux et de techniques (dont certaines se retrouvent ailleurs, comme dans l'archipel de l'Amirauté) ni l'histoire «maritime et coloniale » à laquelle elles doivent leur nom ne suffisant à faire des îles Salomon une "province stylistique ", il faut ainsi en chercher les indices dans des parentés de goûts, de sentiments, de sensations ou de postures reliant les insulaires à distance et autorisant entre eux des échanges qui ne furent pas constamment belliqueux. Au-delà de leurs discordances, les titres des deux expositions en posent l'un et l'autre le principe. Comme l'explique le maitre d'œuvre de la première, Crispin Howarth, dans l'introduction du catalogue $(2011: 22)$, varilaku est un terme de la lagune de Marovo désignant la qualité nécessaire à la chasse aux têtes, la témérité - c'est aussi, ajoutera-t-on, le nom, ironique ou non, d'un lieu-dit de Roviana d'où partaient les expéditions contre Marovo (Gina, 2003: 2 ; Liligeto, 2006 : 59-61) - avec cela que cette chasse toucha surtout le centre de l'archipel et ne peut guère caractériser ses extrémités est et ouest. Le titre de l'exposition parisienne reprenait celui d'un récent article de Sandra Revolon, commissaire de cette manifestation au côté de Magali Mélandri, à propos de l'usage des nacres et de leurs irisations magiques, censées "imiter la nature pour reproduire le monde " (Mélandri et Revolon 2014: 149, Revolon 2012), analyse à l'évidence fondée pour beaucoup d'objets des îles du centre et de l'est, moins opérante pour ceux, non moins nombreux, surtout dans les îles occidentales, dépourvus de ces inclusions de nacre.

Malgré leurs efforts respectifs, aucune des deux expositions ne pouvait aborder de front cette épineuse question de l'unité des expressions plastiques de l'archipel, puisque nil'une ni l'autre n'en livraient un aperçu suffisamment représentatif. Celle de Canberra s'attachait aux îles Salomon d'avant leur partition coloniale de 1899, telle que les représentait par exemple la carte servant de frontispice au livre de Guppy (1887), courant de Buka à Santa Ana, et plaçant encore Santa Isabel, Choiseul et les Shortland " under German influence ". Forte d'une soixantaine d'objets, elle ne faisait aucune mention des "enclaves polynésiennes " aujourd'hui rattachées à l'archipel, ni même de la province de Temotu (Santa Cruz, Tikopia et Vanikoro principalement), toutes absentes de la carte ouvrant le catalogue. Rassemblant deux cents œuvres et documents (certains seulement dans le catalogue, d'autres présentés mais non reproduits), l'exposition parisienne comportait quant à elle des objets de Rennell, Bellona et des Santa Cruz, faciles à situer sur la "carte linguistique des îles Salomon " illustrant l'excellent article consacré par Angela Terrill aux "langues des îles Salomon, reflets des interactions sociales ", carte s'arrêtant - et la sélection d'objets aussi - à une « Papouasie Nouvelle-Guinée " laissée en blanc au nord-ouest de la frontière de 1899, comme si ni Bougainville ni Buka n'avaient eu d'importance dans l'histoire des arts salomonais ${ }^{2}$. C'était du même coup priver le public du gros millier de spécimens de ces îles conservé au musée du quai Branly et sur lequel Nicolas Garnier a récemment rédigé une très remarquable et utile "note de recherche" (2013), et omettre un des trois "cultural districts " acceptés par la plupart des spécialistes précités. Si cette manifestation avait eu, comme il arrive dans ce musée, un caractère diplomatique impliquant les autorités d'Honiara, cette sélection d'objets limitée aux frontières politiques actuelles y aurait trouvé sa justification, mais tel n'était pas le cas. Dans leur introduction, M. Mélandri et $S$. Revolon n'explicitent nulle part leur choix, et les lecteurs peuvent s'étonner de se voir rappeler, dans l'exposé de Peter Sheppard qui suit, qu'à la faveur du dernier maximum glaciaire,

2. Une autre carte (pp. 18-19) mentionne cependant Bougainville et Buka, mais pour illustrer la contribution de P. Sheppard consacrée à l'archéologie de l'archipel qui souligne l'importance du site de Kilu, à Buka, datant d'environ 27000 ans avant notre ère. Classiquement, archéologues et spécialistes des arts du Pacifique désignent ces îles sous le nom de "nord-ouest des Salomon ", d'où un quiproquo permanent, les "Western Solomons " des cartes politiques recoupant ce qu'ils nomment quant à eux «Salomon centrales » (voir par exemple Kaufmann, 1993 : 560-563). 
" théoriquement toutes accessibles à pied, les îles principales de Buka à Guadalcanal ont pu être occupées dès le pléistocène supérieur»

offrant ainsi un continuum à prendre en compte, au moins du point de vue de l'archéologie, mais aussi pour l'étude des arts et des techniques de date plus récente.

\section{Éclairages et zones d'ombre}

S'agissant d'arts, on signalera d'abord la belle attention portée aux objets par chacun de ces catalogues, avec d'abondantes photographies en pleine page, maints détails montrés plus grands que nature venant conforter ce sentiment de "luxe " et d' "élégance" mis en avant par D. Newton et D. Starzecka, et des textes s'attachant principalement à replacer les œuvres dans leur contexte. Dans Varilaku, outre la contribution précitée de D. Waite sur le "langage corporel » et un avant-propos de David Attenborough rappelant qu'à côté de la réputation de raffinement et de férocité faite aux Salomonais par les Occidentaux, les combats de la guerre du Pacifique, à Guadalcanal et ailleurs, ont montré de quoi étaient capables ces derniers dans les mêmes registres, on trouvera, sous la signature de $\mathrm{Cr}$. Howarth, une introduction générale ("Land, People and spiritual Environment», pp. 12-31) ne cachant rien des exactions coloniales, et un catalogue thématique, envisageant successivement les sculptures anthropomorphes ("Bodies, abstraction and realism ", pp. 48-63), les parures et les ornements de prestige ("Objects of Authority, Enhancement and Enchantment ", pp. 64-85), les objets à caractère sacré ou magique ("Objects of contained Power ", pp. 86-93), et pour finir, les figures de proue et les autres sculptures liées à la navigation et aux hangars à canots, à leur décoration et à leurs reliques ("Beyond Control: Sea, Air and Spirit Beings", pp. 94-119).

À l'exception de deux d'entre elles et de quelques photographies anciennes en mains privées, toutes les ouvres retenues provenaient d'institutions publiques australiennes, et beaucoup n'avaient jamais été reproduites ou publiées avec tant de soin. Parmi les pièces inédites, généralement de grande qualité, il faut mentionner la longue planche ornementée $(2,56 \mathrm{~m})$, provenant sans doute d'une maison des hommes de Buka, retrouvée par Cr. Howarth dans les réserves d'un musée tasmanien, objet sans équivalent dans les collections publiques d'Australie sinon d'ailleurs. Les légendes précisant toujours les dates de collecte ou d'entrée dans les catalogues permettent de distinguer les acquisitions anciennes des plus récentes, judicieux principe évitant d'avancer des datations " impressionnistes " impossibles à véri- fier du fait des matériaux mis en œuvre: on ne peut présenter tel brassard de Makira en fossile de bénitier acquis en 1892 par le musée Victoria de Melbourne (p. 11 et 65) que comme antérieur à cette date, sa datation géologique n'étant ici d'aucun intérêt et les techniques de fabrication échappant à toute chronologie précise. Certains achats récents de la National Gallery of Australia complètent les connaissances en matière de circulation des objets sur le marché. Ainsi, le poteau de hangar à canots de Santa Ana présentant deux personnages superposés (p. 112), acquis en 2006, provient de la même "collecte " qu'une pièce figurant dans l'exposition parisienne $\left(\mathrm{n}^{\circ} 78\right.$, p. 171), elle aussi passée entre les mains du marchand parisien Charles Ratton, après celles de son concurrent et ami Ernest Ascher, les deux sculptures ayant été très probablement achetées sur place par le "chercheur indépendant" et photographe Hugo Adolf Bernatzik (1936: 6) durant son séjour de 1932 chez le "roi blanc" de l'île Henry Küper. Ces deux poteaux furent du reste identiquement raccourcis à Paris, à la demande du même Ratton.

On ne peut s'attarder ici sur l'excellente sélection d'objets opérée par Cr. Howarth, y compris pour des œuvres "naturalistes " tardives, qu'on dirait volontiers "de commande" ou "d'art colon ", mais dont D. Waite (p. 39 sq.) et luimême défendent l'intérêt, voire le caractère traditionnel, et sur certaines desquelles N. Garnier, dans la «note " précitée (2013 : 17, à propos du sculpteur Genu), ajoute d'utiles compléments. Marquant un égal souci de qualité, les objets rassemblés dans l'exposition parisienne provenaient pour un tiers (44) de collections publiques françaises, et pour le reste de musées étrangers et de prêteurs privés, marchands ou non, "provenances » trop diverses pour proposer mieux, dans les légendes, que des datations conjecturales. L'Éclat des ombres, le catalogue qui les présente, réunit une trentaine de contributions de taille et d'ambition variées, impossibles à énumérer dans ces colonnes, mais dont la liste des signataires, à compléter des noms précités, suggère tout le sérieux et l'importance : David Akin, Ben Burt, Christian Coiffier, Pei-yi Guo, Edvard Hviding, Christine Jourdan, Jari Kupiainen, Géraldine Le Roux, Pierre Maranda, Michael W. Scott, Tim Thomas, D. Waite, Goeffrey M. White, Johanna Whiteley, soit nombre de spécialistes réputés des cultures de l'archipel et de leurs arts.

Hormis l'introduction, les articles de P. Sheppard, d'A. Terrill et l'essai historique de D. Akin et G. M. White sur « les artefacts de la guerre, art, échange et politique pendant la Seconde Guerre mondiale " qui ont une portée générale, la plupart des textes se concentrent sur l'étude des îles orientales (Malaita, Makira, Santa Ana) et de la Nouvelle-Géorgie et de ses environs, reflétant l'état lacunaire des connaissances actuelles et la 
concentration des chercheurs sur des "cultural districts" déjà assez bien explorés. Deux brefs articles de J. Whiteley sont consacrés à des objets de Santa Isabel, J. Kupiainen consacre deux développements à Rennell et Bellona, un autre plus bref, dû à G. Le Roux, s'attache aux monnaies de plumes de Santa Cruz, aucun n'évoque les arts de Choiseul pourtant représentés au catalogue par deux objets, et la sculpture de Guadalcanal est illustrée par deux " porteuses de coupe ", sans commentaire explicatif (voir ci-après celle dont le musée du quai Branly a aimablement autorisé la reproduction dans le $J S O$ ), tandis que les sociétés de cette île ne se trouvent mentionnées qu'à propos de cuisine, dans le texte que consacre Chr. Jourdan aux "cultures alimentaires" du centre et de l'est de l'archipel.

Si l'histoire des arts, et même l'ethnologie, des îles Salomon comptent d'importantes «zones grises ", il subsiste sur certaines d'entre elles quelques lueurs fournies par les vieilles explorations occidentales, méritant au moins d'être rappelées au public de ce pays-ci. Le temps n'est plus où un rédacteur des Cahiers coloniaux de 1889 (Mager 1889 : 301-302) pouvait s'écrier :

"Les îles Salomon sont françaises par leur nom, françaises par leur histoire. [...] Le 31 janvier 1887, les journaux de Paris, le National entre autres, publièrent cette information officieuse: "Une note diplomatique affirmant les droits de la France sur l'archipel Salomon a été remise à l'Allemagne." Devons-nous renoncer à tout jamais au groupe septentrional, à la baie de Choiseul, au port Praslin, à la baie de Mille Vaisseaux située (sic) sur les côtes des îles Choiseul et Isabelle ? Pourquoi ne pas prendre possession des trois îles méridionales : Malaïta, Guadalcanar, SaintChristophe, qui sont restées en dehors de la sphère d'action que l'Allemagne s'est assignée ?"

Parmi les arguments énumérés par ce rédacteur - par exemple, nourrir la Nouvelle-Calédonie dont les îles Salomon seraient "le salut ", et " grâce à leur fertilité ", également " le grenier " - l'un des moins discutables est qu'elles furent:

«baptisées et explorées par des navigateurs français, par Bougainville, Surville, d'Entrecasteaux, Dumont d'Urville. »

L'exposition parisienne réunissait quelques pièces collectées par l'équipage de ce dernier, passé à Malaita et à Santa Isabel en novembre 1838, mais présentées de façon quelque peu disparate. Était attribué à ce voyage un "avant de pirogue " conservé au musée national de la Marine ( $\mathrm{n}^{\circ} 65$ du catalogue, p. 137, inv. 39 EX 7 , ce même musée possédant un autre spécimen de même origine, 39 EX 6, très érodé mais avec un personnage aux mains levées à mettre en relation avec l'analyse que fait $\mathrm{D}$. Waite des boucliers offrant cette posture dans le catalogue



Figure 1. - « Bol cariatide. Île de Guadalcanal, côte nord. Fin du XIX ${ }^{\mathrm{e}}$ siècle. Bois teinté (Alstonia scholaris), nacre, résine, graines $64 \times 26 \times 22 \mathrm{~cm}$. Legs Édouard-Victor Saint-Paul. Musée du quai Branly, inv. 71.1977.42.1» (C) Courtesy musée du quai Branly)

de Canberra, p. 41, ou encore avec le bouclier du musée du quai Branly, inv. 70.2003.4.1, p. 142), évidemment collecté à Santa Isabel, le catalogue ne le signalant pas. Tel était également le cas du pendentif en conus (Musée du quai Branly inv. 72.56.193, non reproduit) et d'un autre en tridacne et fibres végétales teintées (Musée du quai Branly, inv. 72.56.196, nº 90 du catalogue, p. 190), eux aussi à restituer à Santa Isabel, pour des raisons qu'on verra plus loin. À l'inverse, un étui à chaux en bambou gravé (musée du quai Branly inv. 72.56.169, $\mathrm{n}^{\circ} 79 \mathrm{du}$ catalogue, p. 176) est justement donné comme de la même île, sans autre indication, alors que la base de données du musée attribue à la même circumnavigation sa collecte, celle d'un autre spécimen très proche $(72.56 .168 .1-2$, ne provenant manifestement pas de Vanikoro), et d'une petite dizaine d'objets témoignant du passage de L'Astrolabe et de La Zélée sur ces côtes des îles Salomon. Des pièces analogues sont conservées dans plusieurs musées régionaux, dans le Nord de la France en particulier (S. Jacquemin, 1997 : 46-47), et une vitrine n'aurait pas été de trop pour signaler l'intérêt de cette collecte.

En même temps que se préparait ou que se tenait cette exposition du musée du quai Branly, des antiquaires spécialisés de la place de Paris proposaient des objets acquis sur les mêmes rivages par l'ingénieur hydrographe de l'expédition, Adrien Vincendon-Dumoulin, ensuite conservés dans la résidence familiale, puis dispersés en vente publique en mai 2003 (Gros-Delettrez 2003). L'un 
de ces antiquaires avait quelque raison de présenter ensuite une figure de proue nguzunguzu issue de cette vieille collection comme

"one of the earliest examples ever collected by European explorators. " (Meyer, 2011, $\mathrm{n}^{\circ} 3$ )

Haut de $22 \mathrm{~cm}$, cet objet provient très probablement de Santa Isabel, la dernière île de l'archipel où aient accosté ces navigateurs. Ces exemples, auxquels on pourrait ajouter des dizaines d'autres, suggèrent qu'en France la situation de " dispersion » et de défaut d' "investigation » des collections historiques océaniennes jadis constatée par Marie-Charlotte Laroche (1945: 53 sur les collections "Dumont d'Urville») il y a 70 ans a laissé de profondes séquelles, en dépit des efforts récents pour y remédier. Du même point de vue, on peut regretter que, malgré l'abondance et la précision de leurs observations faites à terre, et jusqu'à l'intérieur des maisons cérémonielles, les récits publiés de la même expédition (Dumont d'Urville [Vincendon-Dumoulin et a.] 1843 : 21-87, 104-113, 295-304), sans parler de versions inédites pouvant mériter l'impression, n'ont jusqu'à présent donné lieu à aucune étude du point de vue de l'ethnographie ou de l'histoire des arts des îles Salomon, pour les confronter aux objets subsistant aujourd'hui de ce périple.

Quoique Dumont d'Urville ait écrit lui-même (1835: II, 155) que l'expédition de Surville, qui fit escale en octobre 1769 à Santa Isabel, avait rapporté «les seuls documents un peu étendus que l'on possède" sur l'archipel, ses officiers la créditant plus tard d'avoir légué "sur ces îles les notions les plus complètes que nous en ayons " (1843: 100), le périple qui mena le Saint-Jean Baptiste de Pondichéry à Callao, le faisant s'arrêter notamment aux îles Salomon et à l'extrême nord de la Nouvelle-Zélande - quelques jours après la première visite de Cook -, n'est pas mentionné dans le texte du catalogue, le nom du navigateur ne figurant que sur la carte de Fleurieu (1790) reproduite pp. 46-47. De cette expédition secrète, mêlant missions d'exploration, de renseignement et de commerce interlope - avec l'appui discret de Choiseul, secrétaire d'État à la marine, et de la haute banque, la famille Law de Lauriston en particulier - subsistent les copies d'au moins quatre journaux de voyage différents en français, certains accompagnés de dessins, et des versions en castillan, destinées à obtenir des autorités espagnoles la mainlevée sur le navire dont elles avaient ordonné la saisie au Chili (Kelly, 1967). Ces mêmes autorités s'alarmèrent tellement des menées françaises venues à leur connaissance qu' elles lancèrent aussi- tôt une de leurs dernières missions d'exploration, celle du San Lorenzo (Corney, 1903 : XLI sq.).

Les apports des circumnavigations concomitantes de Bougainville et de Cook rejetèrent dans l'ombre celle de Surville, dénuée de prétention scientifique et fâcheusement conclue par la mort du capitaine et de son "invité " maori aux abords du Chili, puis l'immobilisation du navire pendant trois ans (1770-1773). Il fallut attendre une décennie de plus pour voir publiés les premiers extraits de journaux, en appendice de ceux du périple de Marion Dufresne (Rochon, 1783 : 251-290), dans un contexte de tension francobritannique lié à la guerre d'Indépendance américaine déterminant quelques années plus tard la publication d'extraits plus amples, à l'appui de revendications territoriales sans détour (Claret de Fleurieu 1790 : 93-154, Laborde 1791 : II, avertissement 42-62, texte 1-115). Plus de deux siècles s'écouleront avant la première édition intégrale en français de l'un de ces journaux, celui du lieutenant Pottier de l'Horme (Morgat, 2004), suivie peu après par une évocation assez sommaire de ce voyage due à l'historien néozélandais (Dunmore, 2008) l'ayant pourtant le plus précisément étudié (Dunmore, 1981).

C'est encore en Nouvelle-Zélande, voilà un siècle (McNab, 1914), que fut traduit et commenté un autre de ces journaux, celui de Pierre Monneron, subrécargue du Saint-Jean Baptiste et écrivain du bord, lié aux Choiseul et à la haute banque, chargé des missions secrètes et des négociations avec les autorités espagnoles, arrivé à Paris dès 1771 en compagnie de Lova Sarega ${ }^{3}$, "petit sauvage enlevé de son pays " lors d'un affrontement survenu à Santa Isabel le 14 octobre 1769 , et auquel ces journaux sont redevables de leurs précisions ethnographiques sur cette partie des îles Salomon, dénommée par Surville "Arsacides » (c'est-à-dire "Assassins »). D'autres chercheurs néo-zélandais ont depuis lors scruté les documents subsistant de ce périple pour ce qu'ils rapportent utilement des Maori (par exemple Ollivier et Hingley, 1982). Mais ces textes continueront d'être ignorés des historiens (tout récemment, West-Sooby, 2013: 5, 24, 35, mentions insignifiantes) et des ethnologues ou commissaires d'exposition tant qu'ils n'auront pas été édités avec l'ampleur et la rigueur nécessaires. L'entreprise n'est pas simple: les dessins associés aux copies françaises et espagnoles du journal de Monneron sont probablement à restituer à Pottier de l'Horme, lequel, resté au Chili, ne put lire la relation du voyage de Bougainville qui « informe " ou déforme la rédaction de Monneron (fait déjà observé par Claret de Fleurieu, 1790 :

3. Aucune étude ne semble avoir été consacrée à Lova Sarega, contrairement à Omaï et à d'autres insulaires du Pacifique " dépaysés " par des navigateurs occidentaux au cours des mêmes années. Dans les commentaires élogieux qu’il fait de sa "probité " et de ses " heureuses dispositions ", Monneron en parle au présent, comme pour le recommander à d'autres protecteurs français, et sa rédaction est signée et datée de " Paris, le 4 octobre 1771 ». Le destin du premier "informateur ethnographique " des îles Salomon arrivé en Europe reste à éclairer. 




Figure 2. - Claret de Fleurieu, Découvertes des François en 1768 et 1769 au Sud-Est de la Nouvelle Guinée.. 1790, planche vir. (C) archives de l'auteur)

104 note), elle-même plus riche en renseignements obtenus de Lova Sarega, etc. Pour l'heure, on ne peut qu'emprunter de brèves citations aux textes déjà édités pour suggérer l'intérêt du témoignage ethnographique qu'ils relaient et qu'on pourrait presque qualifier "de première main ".

On s'en tiendra donc ici à deux exemples en rapport avec ces expositions. Pour ce qui est des ornements corporels masculins, celle de Paris présentait un "pectoral » en bénitier fossile (p. 190 du catalogue, MQB inv. 72.56.196) collecté lors de l'escale de Dumont d'Urville à Santa Isabel, et qu'il n'est peut-être pas exact de qualifier de pagosia, terme propre à la lagune de Roviana (Nagaoka, 2011 : 189 et 450). Les compagnons de Surville avaient décrit en ces termes

Figure 3. - Laborde, Histoire abrégée de la Mer du Sud, 1791, tome II (@ archives de l'auteur)








Figure 4. - " Pendentif pagosia, îles Salomon occidentales, début du XIx ${ }^{\mathrm{e}}$ siècle. Coquillage (Tridacna sp.), fibres végétales teintées. Collecté par Jules-Sébastien César Dumont d'Urville, second voyage de l'Astrolabe et de la Zélée, 1837-1840. Paris, musée du quai Branly inv. 72.56.196» (C) Courtesy musée du quai Branly)

ce type d'objet apparemment attesté aussi à Florida (Waite, 1987: 68 et pl. 15) :

"Quelques-uns suspendent aussi à leur col une espèce de peigne d'une pierre blanche à laquelle, suivant le rapport de Lova Sarega, ils attachent un très grand prix. » (Fleurieu, 1790 : 128, sans doute d'après Labé)

« Ils portent au-dessus du coude un cercle, et au cou un ornement qui a la forme d'un peigne ; il est fait avec une pierre blanche qu'ils estiment beaucoup, selon ce que nous dit le jeune sauvage que nous avons pris. Ils ont aussi plusieurs sortes de bracelets. » (Laborde, 1790, II : 63-64, d'après Monneron $\left.{ }^{4}\right)$

Parmi les chercheurs contemporains, Bill Evans (2005 : 240) est le seul à s'être intéressé au dessin de "bouclier de jonc long de 3 pieds, rerero" accompagnant plusieurs versions de ces journaux, qui en décrivent aussi les principaux usages :

" Ils ont, pour se garantir des flèches, un bouclier fait de rottin fendu, tressé comme nos ouvrages en osier plein : une des faces est garnie de deux espèces d'anses ou poignées pour y passer le bras. Ils s'en couvrent le dos \& la tête, lorsqu'ils sont assis dans leurs bateaux, $\&$ s'en servent aussi de parapluie. Quelques-uns de ces boucliers sont ornés aux quatre coins de houpes ou floccons faits d'une espèce de ruban de paille rouge $\&$ jaune » (Fleurieu, $1790: 130$, ici d'après Monneron ${ }^{5}$ )

Comme le suppose avec vraisemblance B. Evans, ces boucliers à sommet bilobé, paraissant avoir " a more complex structure than the later shields", furent remplacés au XIX ${ }^{e}$ siècle par des objets de fabrication plus simple, en enroulements elliptiques de vannerie ou en longs rectangles d'écorce renforcés par une armature convexe, tel celui, sans décor, recueilli lors de l'escale de Dumont d'Urville à Santa Isabel que conserve le musée du quai Branly (Inv. 72.84.358). Si, ainsi qu'il semble, l'invention de ce type de bouclier convexe (avec des dimensions comparables à celles de leurs prédécesseurs, "trois pieds " environ) doit être assignée à la même île, il ne serait pas incongru de mettre en relation les figurations symboliques ornant les plus prestigieux d'entre eux (catalogue de Paris pp. 142 et 140, Musée du quai Branly inv. 70.2003.4.1 et 71.1887.67.9, ce dernier à comparer avec celui du catalogue de Canberra, pp. 80-81, Australian Museum E 25422), représentant sans doute des personnages aux bras levés, avec les motifs non moins stylisés dont s'ornent les pièces de tapa qui partaient de cette île en direction de la Nouvelle-Géorgie ou d'autres lieux d'échange de l'archipel. L'un des mérites de l'exposition parisienne et de ses commissaires est d'avoir pris en compte et prolongé les recherches récentes sur ces tapas et leurs décors (notamment Richards et Roga, 2005), introuvables dans l'exposition et le catalogue de Canberra, faute de place probablement.

On trouvera dans L'Éclat des ombres de nombreuses autres avancées rendant cet ouvrage indispensable. Beaucoup concernent des objets peu connus, tels les bâtons de danse en forme d'oiseau décrits par P. Maranda (178-179), ou souvent envisagés jusqu'à présent hors de toute perspective historique, à l'instar des «lances cérémonielles" de Bellona et Rennell, dont J. Kupiainen (pp. 50-55) relate l'invention « vers le milieu du XIX ${ }^{e}$ siècle $"^{6}$. C'est l'introduction de cette perspective historique, animant l'organisation même du catalogue parisien, entre "transformations " (pp. 49-144) et "permanences " (pp. 145-209), qui constitue sans doute le trait

4. C'est ce que montre la traduction de McNab (1914: 256) : "They wear a bracelet above the elbow, and round the neck an ornamant which resembles a comb, made of a white stone, which they hold in great esteem, according to the young savage we captured. They possess several kinds of bracelets. " Pottier de l'Horme n'a pas décrit cet objet. Souvent cité comme riche d'informations de ce genre, ce qui est publié du journal de Shortland (Phillip, 1789 : 198) ne consacre que trois phrases vagues aux ornements corporels des Salomon.

5. "To guard themselves against arrows, they carry a shield made of tantan cane" etc. (McNab, $1914: 257)$. La description de Pottier de l'Horme (Morgat, $2004:$ 131-132) est plus diserte.

6. Voir néanmoins Monberg (1991 : 174) qui écrivait déjà : "The shape of this spear was "invented" fairly recently ", etc. 


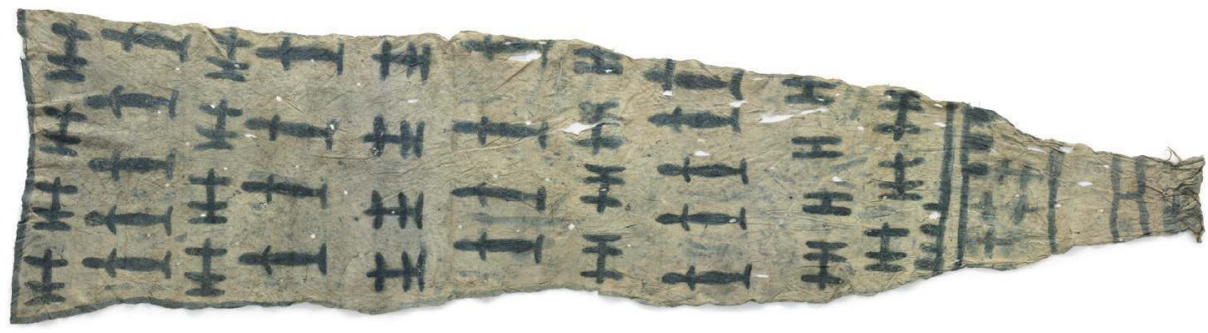

Figure 5. - «Étoffe d'écorce battue, pohe. Île de Santa Isabel. Début du XXe siècle (?). Liber d'écorce battu, colorants naturels. 255 x $90 \mathrm{~cm}$. Paris, musée du quai Branly, inv. 72.1992.0.13» (C Courtesy musée du quai Branly)

le plus neuf de ces deux expositions, même si aucune des deux n'évoque ce qui est advenu de l'archipel au sortir de la guerre du Pacifique, spécialement au cours des dernières décennies marquées par des tragédies à répétition, tant du côté de l'État des îles Salomon que de celui des «îles Salomon du Nord-Ouest ", anciennement allemandes, puis sous mandat australien, avant de revenir à la Papouasie Nouvelle-Guinée : là, des dizaines de milliers de déplacés et des centaines de victimes lors de conflits "ethniques " à Guadalcanal et ailleurs, ici, dix ans de guerre civile à Bougainville (1988-1998) autour de l'immense mine de cuivre de Panguna, entre 15 et 20000 morts selon les estimations des autorités australiennes, dont les troupes sont intervenues sur ces deux « théâtres d'opération ». Ces tragédies n'ont évidemment pas de rapport direct avec l'histoire des arts océaniens, leurs blessures sont toujours ouvertes, les armes continuent de parler, et il était légitime de la part des responsables des expositions de Canberra et de Paris de se concentrer sur les objets à montrer et les cultures anciennes qu'ils représentent et dont le public d'éducation européenne reste peu familier. Pourtant, peuton oublier les Salomonais d'aujourd'hui ?

\section{" Ethnicisation » et histoire des arts}

Un des articles les plus éclairants de L'Éclat des ombres, "La métaphysique des particules» (pp. 172-176), dû à M. W. Scott, s'attache aux divers usages de la chaux à Makira et alentours, et particulièrement à sa vertu de transmettre à qui s'en pare le visage ou le corps des pouvoirs magiques, mena, terme arosien dérivé du mana océanien. Or, note-t-il (pp. 174 et 176),

" la peinture corporelle à la poudre de chaux est aujourd'hui devenue emblématique de la coutume (kastom). [...] La particule mena de Makira, dans le contexte actuel d'ethnicisation croissante des identités insulaires et régionales, peut être utilisée contre les autres mais reconnaît et protège les siens. »

Depuis une décennie au moins, cette " ethnicisation » est désignée par de nombreux observateurs,

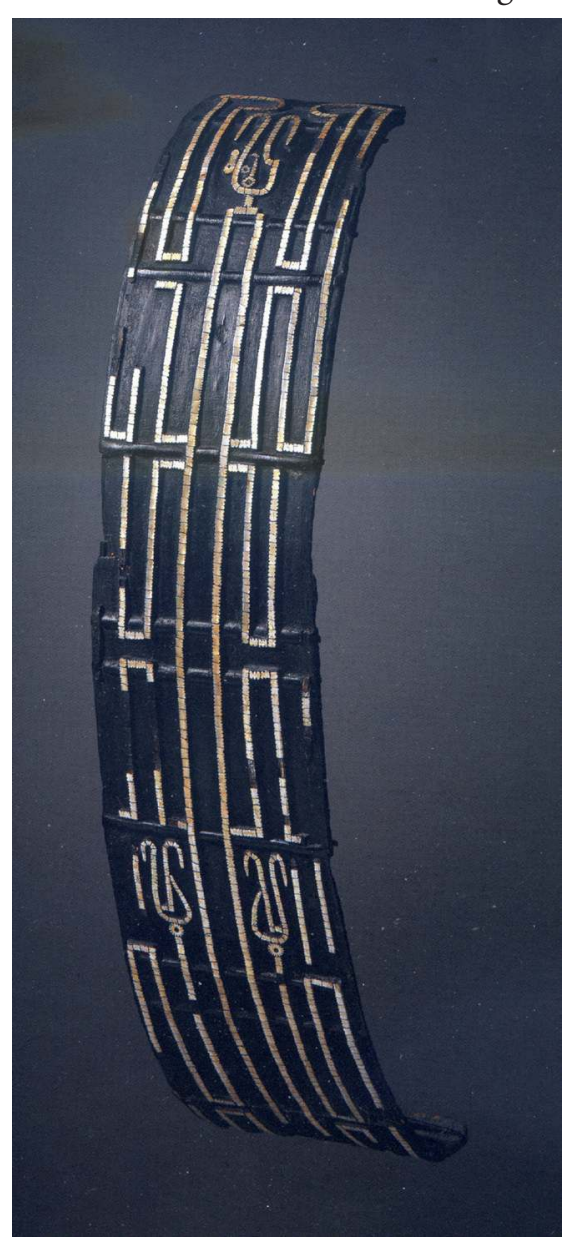
ceux de l'Union européenne et des Nations unies en particulier (par exemple UDNP 2004 : 5, Olyan et a. 2010 : 5, voir aussi l'enquête de Wood, 2014), comme l'un des risques majeurs encourus par les habitants de l'archipel, également menacés par la "vulnérabilité climatique", le déboisement, la pollution et les expropriations liées aux gigantesques projets miniers en cours, à Guadalcanal pour l'or, à Santa Isabel et dans tout le sud-est de Choiseul pour le nickel, à Rennell pour la bauxite, etc., les insulaires des Salomon restant victimes de la même convoitise pour les métaux qui avait attiré les premiers Occidentaux chez eux.

Figure 6. - « Bouclier. Île de Santa Isabel. XIX ${ }^{\mathrm{e}}$ siècle. Écorce, résine (noix de Parinarium), nacre. $87,5 \times 23 \mathrm{~cm}$. Ancienne collection Hunter. Paris, musée du quai Branly, inv. 70.2003.4.1" (C) Courtesy musée du quai Branly) 
Figure 7. - « Île de Rennell, village de Niupani. Début du xx siècle. Bois 192 x 12,5 x $3 \mathrm{~cm}$. Collecté par Monique de Ganay, expédition de La Korrigane, 1935. Musée du quai Branly, inv. 71.1961.103.45» (C) Courtesy musée du quai Branly)

Que «l'ethnicisation» soit à considérer comme une résultante d'autres crises actuelles (économique, politique, environnementale...) frappant l'archipel ou comme un facteur quasiment indépendant - remontant à l'histoire même de la colonisation et à ses injustices selon John Houainamo Naitoro (2000:3), et pouvant orienter la politique vers un certain type de prophétisme millénariste, comme $\mathrm{M}$. W. Scott l'a montré récemment (2014) pour les îles Salomon orientales -, les ethnologues et les historiens des arts océaniens ne peuvent que s'en inquiéter, sauf à se satisfaire d'une " anthropologie inutile", selon la formule de Georges Guille-Escuret, ou d'une érudition décorative au service de ce que l'esthétique a de plus frivole. De l'étude des cultures traditionnelles des îles Salomon, à l'approfondissement de laquelle ces catalogues auront contribué chacun à leur manière, on doit attendre non seulement qu'elle apporte ses lumières sur les «zones grises » des sociétés et des mours du temps jadis, mais aussi qu'elle contribue à dissiper les obscurantismes du temps présent, cette variante du racisme qu'on nomme l'"ethnicisme" n'étant pas le moindre. Dans ce contexte, même la question de la continuité, voire de l'unité, des traditions artistiques de l'archipel évoquée au début de ce développement a une dimension politique dont les spécialistes doivent prendre toute la mesure.

S'exprimant en tant que directeur de la Bougainville Cultural Foundation, le prestigieux leader indépendantiste Moses Havini (né en 1947 à Buka, décédé à Sydney en mai 2015) a formulé des propositions pleines de raison sur les relations à établir entre les musées occidentaux et les Salomonais, en vue de transferts de connaissances allant dans les deux sens, avec ce qu'ils supposent de " meaningful dialogue " et de "co-operation" (2003: 125). Entre 2005 et 2010 (Bolton et al., 2013, voir JSO 138-139), les océanistes du British Museum ont mené à bien le Melanesian Art Project reposant sur les mêmes principes, et consistant notamment à inviter des "experts locaux " à examiner les collections londoniennes. Pour ce qui est des îles
Salomon, un ouvrage collectif (Burt et Bolton 2014) a pu réunir pour la première fois des éclairages de première main dus à des insulaires, Jackson Gege (Clan valuables of Guadalcanal), Evelyn Tetehu (Some family treasures of Santa Isabel), Michael Kwa'ioloa (Traditional money and artefacts in Malaita), Salome Samou (Santa Cruz feather-money: Its demise and revival). Cette intelligente politique de recherche et de partage des savoirs, qui contribue évidemment à la lutte contre l'«ethnicisation", attend d'être mieux développée à Canberra et à Paris, après les belles et utiles expositions qui y ont été présentées.

\section{BIBLIOGRAPHIE}

Bernatzik Hugo Adolf, 1936. Owa Raha, Wien-Leipzig-Olten, Bernina Verlag.

Bodrogi Tibor, 1959. Oceanian Art, Budapest, Corvina.

Bolton Lissant, Nicholas Thomas, Elizabeth BonsheK, Julie Adams and Ben Burt (ed.), 2013. Melanesia, Art and Encounter, London, The British Museum Press.

BüHLER Alfred, Terry Barrow et Charles P. Mountrord, 1962. Océanie et Australie. L'art de la mer du Sud, Paris, Albin Michel.

Burt Ben and Lissant Bolton (ed.), 2014. The Things We Value. Culture and History in Solomon Islands, Canon Pyon, Herefordshire, Sean Kingston.

Conru Kevin et Deborah Waite, 2012. Trésors des îles Salomon, Paris-Milan, 5 Continents.

Corney Bolton Glanvill (ed.), 1903. The voyage of Captain don Felipe Gonzalez in the ship of the line San Lorenzo... to Easter Island, 17701771, London, Hackluyt Society.

Dumont D'Urville Jules, 1835. Voyage pittoresque autour du monde. Résumé général des voyages de découvertes..., Paris, Tenré. 
- [ADRIEN Vincendon-Dumoulin et al.], 1843. Voyage au pôle Sud et dans l'Océanie..., Histoire du voyage, t. 5 , Paris, Gide.

Dunmore John (ed.), 1981. The Expedition of the St Jean-Baptiste to the Pacific 1769-1770, from the Journals of Jean de Surville and Guillaume Labé, London, The Hakluyt Society.

—, 2009. L'épopée fatale. Le voyage de Surville, Paris, L'Harmattan.

Étude Gros-Delettrez, 2003. Arts Primitifs: Collections Vincendon-Dumoulin et André Malraux, vente le 26 mai 2003 à $14 \mathrm{~h} 30$ salle 10 , Paris, Drouot-Richelieu.

Evans Bill, 2005. Solomon Islands, in Harry Beran and Barry Craig (ed.), Shields of Melanesia, Honolulu, University of Hawaii Press, pp. 237-253.

Claret de Fleurieu Charles Pierre, 1790. Decouvertes des François en 1768 \& 1769, dans le Sud-est de la Nouvelle-Guinée. Et Reconnoissances postérieures des mêmes Terres par des Navigateurs Anglois qui leur ont imposé de nouveaux noms: précédées De l'Abrégé historique des Navigations \& des Découvertes des Espagnols dans les mêmes Parages. Par M.***, ancien Capitaine de Vaisseau, Paris, Imprimerie royale.

Garnier Nicolas, 2013. Les collections de l'île de Bougainville du musée du quai Branly, note de recherche, Paris, musée du quai Branly [http://www.quaibranly.fr/fileadmin/ user_upload/enseignement_et_recherche/ publications_trc/garnier_nicolas_les_collections_de_1_ile_de_bougainville_du_musee_ du_quai_branly_2012.pdf].

Gina Lloyd Maepeza, 2003. Journeys in a Small Canoe. The Life and Times of a Solomon Islander, edited by Judith A. Bennett and Khyla J. Russell, Canberra, Pandanus Books.

Guppy Henry Brougham, 1887. The Solomon Islands and their Natives, London, Swan Sonnenschein, Lowrey and Co.

Havini Moses, 2003. Indigenous perspective on the collection of traditional Bougainville culture and art; past, present and future, $\mathrm{Mu}$ seologia 3, pp. 121-126.

Heanue Siobhan, 2011. Cool Bravado: warrior art from the Solomon Islands, $A B C$ News, 23 février.

Howarth Crispin et Deborah Waite (ed.), 2011. Varilaku. Pacific Arts from the Solomon Islands, Canberra, National Gallery of Australia.

JaCQUemin Sylviane, 1997. Marins et collections : les collectes des expéditions maritimes, in Annick Notter (éd.), La découverte du para- dis. Océanie. Curieux, navigateurs et savants, Paris, Somogy, pp. 41-50.

Kaufmann Christian, Adrienne L. Kaeppler, Douglas Newton, 1993. L'Art océanien, Paris, Mazenod.

Kelly Celsus O.F.M., 1967. Maori and Solomon Islands Drawings from the Surville's Expedition found in Spanish Archives, Journal of the Polynesian Society, 76-4, pp. 459-466.

Косн Gerd, 1969. Südsee. Führer durch die Ausstellung der Abteilung Südsee, Berlin, Museum für Völkerkunde.

LABORDE Jean Benjamin de, 1791. Histoire abrégée de la Mer du Sud, Paris, Didot, 3 volumes.

Laroche Marie-Charlotte, 1945. Pour un inventaire des collections océaniennes en France, Journal de la Société des océanistes, tome 1, pp. 51-57.

Liligeto Wilson Gia, 2006. Babata, our land, our tribe, our people. A historical account and cultural materials of Butubutu Babata, Marovo, Suva, Institute of Pacific Studies.

Mager Henri (éd.), 1889. Cahiers coloniaux de 1889, Paris, Armand Colin.

McNAB Richard, 1914. Records relating to de Surville's Voyage. Monneron's Journal, Historical Records of New Zealand, Wellington, Government Printer, vol. 2, pp. 230-245.

MéLANdri Magali et Sandra Revolon (éd.), 2014. L'éclat des ombres. L'art en noir et blanc des îles Salomon, Paris, Somogy-musée du quai Branly.

Meyer Anthony J. P., 2011. Art of the Solomon Islands-Works of Art from the Pacific Islands, Catalogue tefaf 2011, Paris, Galerie Meyer.

Moloney Kylie, 2011. Varilaku: Pacific Arts from the Solomon Islands, The Journal of $\mathrm{Pa}$ cific History, 46:3, pp. 393-395.

Monberg Torben, 1991. Bellona Island Beliefs and Rituals, Honolulu, University of Hawaii Press.

Morgat Alain, 2004. Le dramatique tour du monde du chevalier de Surville, Paris, Service historique de la Marine-Éditions du Gerfaut.

Musa Helen, 2011. Potent objects of power, desire and beauty, Canberra CityNews, 3-9 mars, p. 23.

Nagaoka Takuya, 2011. Late Prehistoric-Early Historic Houses and Settlement Space on Nusa Roviana, New Georgia Group, Solomon Islands, thèse, University of Auckland 
(https://researchspace.auckland.ac.nz/ handle/2292/9507).

NaItoro John Houainamo, 2000. Solomon Islands Conflict. Demands for historical rectification and restorative justice, Pacific Economic Bulletin, Asia Pacific Press [www.restorativejustice.org/articlesdb/articles/1738].

Newton Douglas, Peter Gathercole, Adrienne L. Kaeppler, 1979. The Art of the Pacific Islands, Washington, National Gallery of Art.

Ollivier Isabel and Cheryl Hingley (ed.), 1982. Extracts from journals relating to the visit to New Zealand of the French ship St Jean Baptiste in December 1769, under the command of J.F.M. de Surville, Wellington, Alexander Turnbull Library. Endowment Trust, National Library of New Zealand.

Olyan Hila and Phoebe Smith, Ruth Stephen, Matthew CASE, 2010. Solomon Islands Risk Assessment Brief, Ottawa, Carleton University (http://www4.carleton.ca/cifp/app/serve. php/1266.pdf).

Phillip Arthur, 1789. The voyage of Governor Phillip to Botany Bay; with an account of the establishment of the colonies of Port Jackson and Norfolk Island; compiled from authentic papers ... to which are added the journals of Lieuts. Shortland, Watts, Ball, and Capt. Marshall, with an account of their new discoveries, London, John Stockdale.

Revolon Sandra, 2012. L'éclat des ombres. Contraste, iridescence et présence des morts aux îles Salomon, Techniques et Cultures, 58, pp. 252-263.

Richards Rhys and Kenneth Roga, 2005. Not Quite Extinct: Melanesian Barkcloth ('Tapa') from Western Solomon Islands, Wellington, Paremata Press.

Rochon Alexis Marie de, 1783. Nouveau voyage à la mer du Sud, commencé sous les ordres de
M. Marion... et achevé après la mort de cet offcier sous ceux de M. le chevalier Duclesmeur..., Paris, Barrois.

Schmitz Carl A., 1971. Oceanic Art. Myth, Man and Image in the South Seas, New York, Abrams.

Scotт Michael W., 2014. Since the 'Ethnic Tension': Prophecy and Political Process in Southeast Solomon Islands, The Edwin Smith Memorial Lecture, Pacific Islands Society of the United Kingdom and Ireland, pp. 1-21 (http://www.pacificislandsuk.org/MScottlecture.pdf).

Starzecka Dorota and Bryan A.L. Cranstone, 1974. The Solomon Islanders, London, The British Museum Press.

STÖHR Waldemar, 1987. Kunst und Kultur aus der Südsee. Sammlung Clausmeyer Melanesien, Ethnologica N. F. 6, Köln, RautenstrauchJoest-Museum.

Und, 2005. Solomon Islands. Peace and Conflict Development Analysis. Emerging Priorities in Preventing Future Violent Conflict, New York, United Nations Development Programme (http://hdr.undp.org/sites/default/files/ hdr2005_mcgovern_and_choulai_33.pdf).

Waite Deborah, 1987. Artefacts from the Solomon Islands in the Julius L. Brenchley Collection, London, British Museum Publications.

West-Sooby John (ed.), 2013. Discovery and Empire the French in the South Seas, Adelaide, University of Adelaide Press [https://www. adelaide.edu.au/press/titles/discovery/discovery-ebook.pdf].

Wood Terence, 2014. Ethnic Identity, Voter Choices and Informal Institutions in Solomon Islands, Canberra, Australian Political Studies Association 2014 Conference Paper (http://papers.ssrn.com/sol3/papers. cfm?abstract_id=2440459). 